International Journal of Forensic Science \& Pathology (IJFP)

ISSN 2332-287X

\title{
A Rare Combination of VACTERL Association with Prune Belly Syndrome- Case Report and Review of Literature
}

\author{
Asaranti $\operatorname{Kar}^{1 *}$, Madhumita Mohanty ${ }^{1}$, Tushar $\operatorname{Kar}^{1}$, Ipsita Dha ${ }^{1}$, Lity Mohanty ${ }^{1}$, Manoranjan Das ${ }^{1}$ \\ ${ }^{1}$ Associate Professor, Pathology, Dept. of Pathology, S.C.B.Medical College, Cuttack, Odisha, India.
}

\begin{abstract}
VACTERL association (any three of vertebral, anal, cardiac, tracheo-esophageal, renal/radial and limb defects) and Prune belly syndrome (complete or partial triad of abdominal muscle deficiency, bilateral cryptorchidism and dilated urinary tract) are cngenital malformations affecting many body systems. These are derivatives of causally nonspecific disruptive events of the developmental field occuring during early blastogenesis. Many of these cases are sporadic in families without much recurrence. These lesions may be associated with other malformations but concurrence of VACTERL with Prune belly syndrome is extremely rare. Routine antenatal check up and ultrasonography can help in diagnosis of most of them. But, many times a detail autopsy examination is needed to detect the variety of malformations and categorise them into specific syndromes. We describe a rare combination of VCTERL with Prune belly syndrome and many associated anomalies of a 28 weeks neonate delivered vaginally by a 37 years second gravida.
\end{abstract}

Keywords: Autopsy; Prune Belly Syndrome; Single Umbilical Artery; VACTERL

\author{
*Corresponding Author: \\ Asaranti Kar, \\ Associate Professor, Pathology, S.C.B.Medical College, Cuttack, Odisha, \\ India. \\ Tel: 09437170442 \\ E-mail:asarantikar@yahoo.co.in
}

Recieved: May, 07, 2014

Published: July, 222014

Citation: Asaranti Kar et al (2014) A Rare Combination of VACTERL Association with Prune Belly Syndrome -Case Report and Review of Literature. Int J Forensic Sci Pathol. 2(5), 43-46. doi: http://dx.doi.org/10.19070/2332-287X-1400013

Copyright: Asaranti Kar Zaba ${ }^{\circ} 2014$ This is an open-access article distributed under the terms of the Creative Commons Attribution License, which permits unrestricted use, distribution and reproduction in any medium, provided the original author and source are credited.

\section{Introduction}

Congenital malformations are a major cause of fetal loss. Most of these fetuses are aborted at an early gestational age. The etiology of these defects may be genetic, environmental or multifactorial. Two such examples of developmental defects are Prune belly syndrome (PBS) and VACTERL association. PBS otherwise known as Eagle-Barrett syndrome or Osler-Parker syndrome is a rare congenital anomaly described most often in male newborns. [1] The term Prune belly syndrome is coined due to the wrinkled, wizened, prune like appearance of abdominal wall and was first used by Williams Osler in 1901. VACTERL/VATER association is typically defined by the presence of at least three of the following congenital malformations: vertebral defects, anal atresia, cardiac defects, tracheo-esophageal fistula, renal anomalies, and limb abnormalities. It was proposed later to include vascular anomalies including single umbilical artery as part of $\mathrm{V}$ in VACTERL. The concurrence of these two syndromes is extremely rare and is gen- erally incompatible with life. The incidence of $1.5 \%$ was reported for the occurrence of PBS with full VACTERL association and $17.5 \%$ for the three components of the VACTERL association. [2] No definite etiology has been proved, but a defect in mesodermal differentiation, in early first trimester, has been suggested for these two syndromes. [3] We report a fetus of 28 weeks with VACTERL association who also had the features of prune belly syndrome and review the literature with clue to antenatal diagnosis.

\section{Case History}

A 37 year old second gravida with previous normal obstetric history and last child birth 7 years back came with complaints of leaking per vaginum $(\mathrm{P} / \mathrm{V})$ and pain abdomen. There was no significant maternal history of any infection, drug intake, hypertension or diabetes mellitus or radiation exposure to mother. The marriage was non-consanguineous. She was undergoing regular antenatal check up in a peripheral hospital. Ultrasonography revealed a single intrauterine live fetus of 28 weeks gestational age having gross abdominal distention with ascitis, thick walled and enlarged bladder, heart pushed to one side, lungs not visualised and one set of limbs traced with oligohydramnios in mother. Fetal heart rate was 144/minute. Placenta was grade II with normal cord insertion. The fetus was delivered by normal vaginal delivery, but died immediately after birth and was sent to Pathology Department for detail autopsy examination.

\section{External examination of the fetus}

Weight of the fetus was $2.4 \mathrm{~kg}$, the crown rump length was $24 \mathrm{~cm}$, crown heel length- $41 \mathrm{~cm}$, head circumference- $29 \mathrm{~cm}$, chest circumference- $30 \mathrm{~cm}$ and foot length was $6 \mathrm{~cm}$. Abdominal girth was $56 \mathrm{~cm}$.Ear cartilage, hair, nails were well developed, palmar and plantar creases were present. The placenta was found to be normal with fully covered membrane and without retroplacental 
Asaranti Kar et al (2014) A Rare Combination of VACTERL Association with Prune Belly Syndrome -Case Report and Review of Literature. Int J Forensic Sci

blood clot or infarction. The abdomen was distended with lax abdominal wall(Fig 1). There was a high placed umbilicus, cord length was $37 \mathrm{~cm}$ with presence of knotting(Fig-1 arrow) and single umbilical artery.(Fig-1 in set)

\section{Congenital malformations}

- Thorax was deformed. (Fig- 1)

- Abdominal cavity was grossly distended. (Fig-1)

- $\quad$ Right foot showed talipes equinovalgus (Fig-1 arrow head)
- Imperforate anus -perineal region had no anal opening (Fig2A)

- Ambiguous external genitalia (Fig-2A).

- Fetal spine was showing kyphosis (Fig-2B ).

- Heart was enlarged and bi-lobed. (Fig- 2C,D)

- Absent gonads.

- $\quad$ Right lung was collapsed (Fig- 3A arrowhead), left lung hypertrophied. (Fig-3A arrow)

- $\quad$ Liver was enlarged in size. (Fig- 3B arrow)

- Enlarged, vertically oriented, thick walled bladder attached

Figure 1. Hugely distended abdomen, deformed thorax and talipes equinovarus of right foot (arrow head), knotting of cord, In set-single umbilical artery.

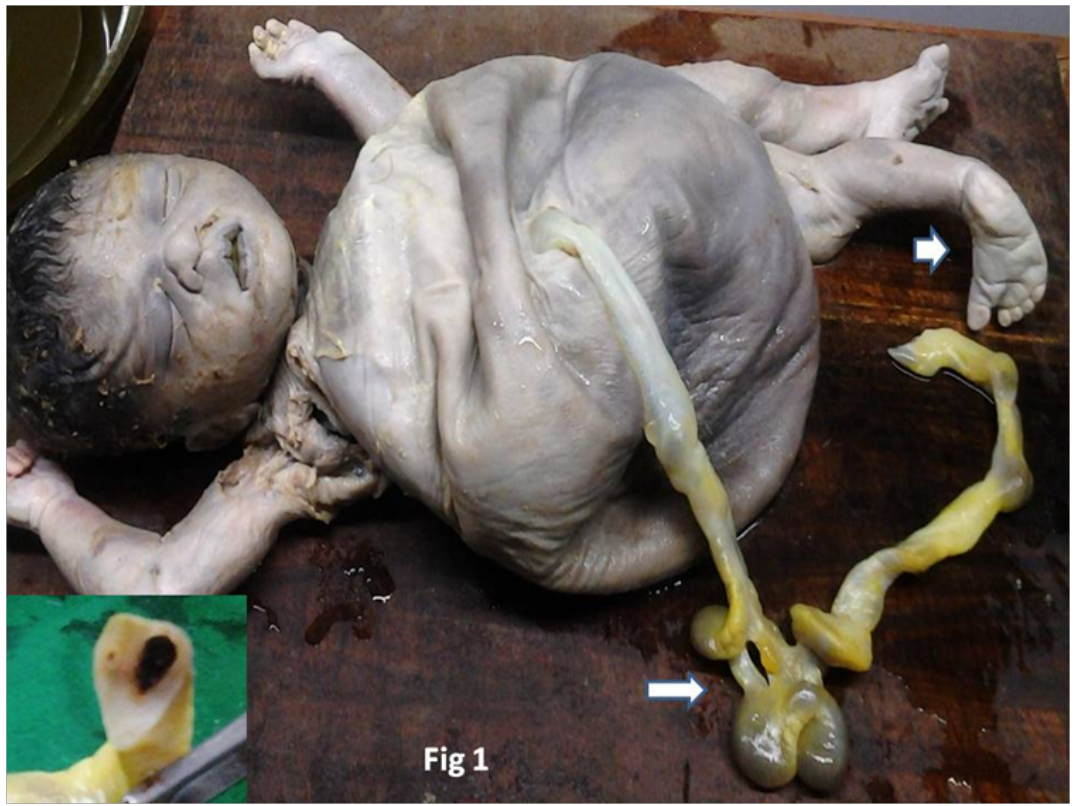

Figure 2. A-Imperforate anus and ambiguous external genitalia, B-Kyphosis of spine,C,D-Enlarged and bi-lobed heart.
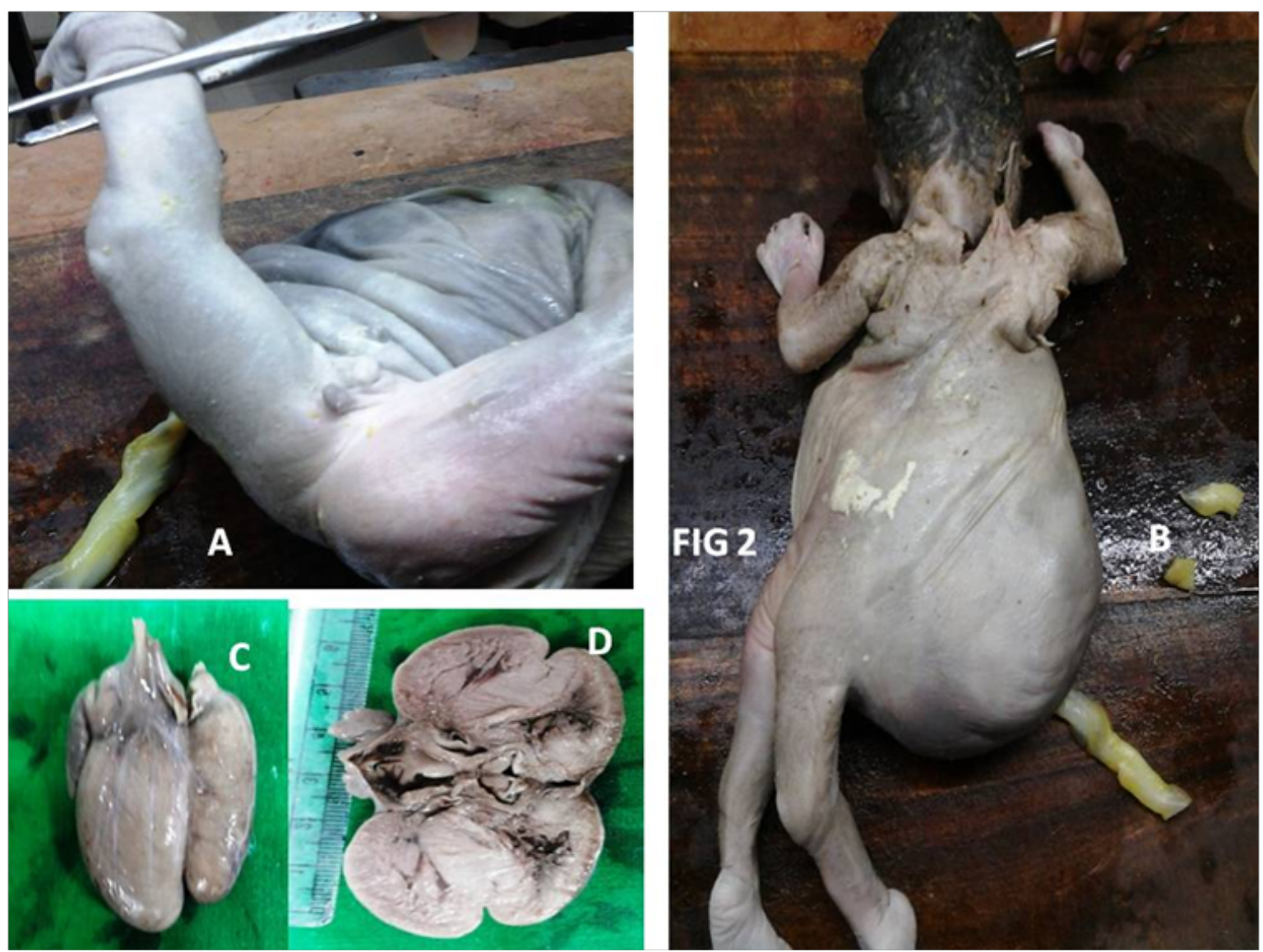
Figure 3. A-A- Collapsed right lung-arrow head, hypertrophied left lung-arrow, B-Enlarged liver- arrow,enlarged, thick walled bladder-arrow head, C-Bilateral tortuous and dilated ureters.

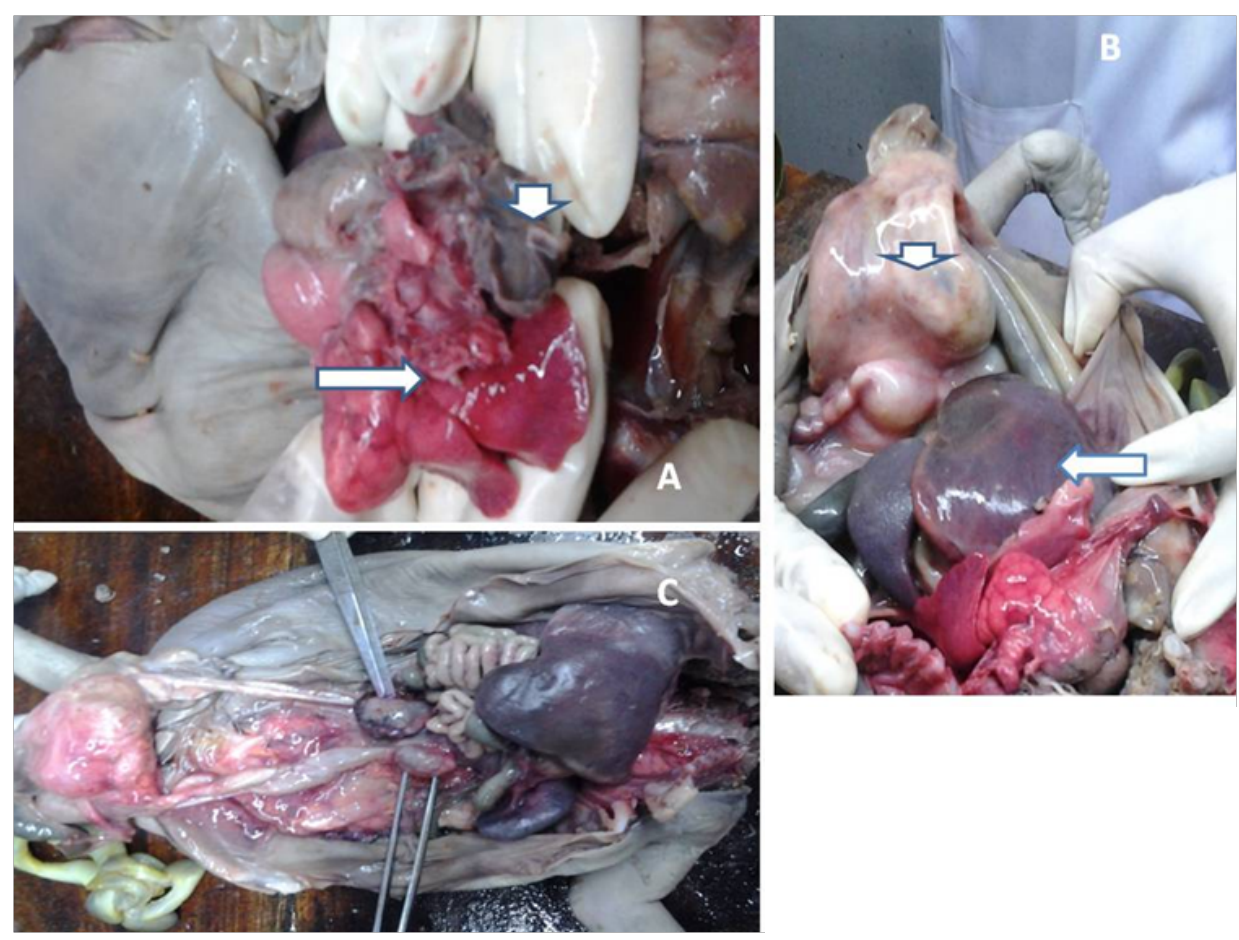

to inner abdominal wall at the site of umbilicus (Patent urachus -Fig-3B arrow head)

- $\quad$ Bilateral tortuous and dilated ureters. (Fig-3C)

On the basis of above anomalies, a diagnosis of VACTERL association (vascular,anal,cardiac,renal,limb anomalies) with PruneBelly syndrome was made.

\section{Discussion}

VACTERL association (VATER syndrome) is a nonrandom cooccurrence of birth defects. It is called association rather than syndrome because the defects are not pathogenetically related but tend to occur more frequentlyt than expected and in general the etiology of association is not clearly known. Incidence of this association is approximately 1 in 10,000 to 1 in 40,000 live births. [4] The incidence of PBS is 3.8cases/1,00,000 live births.[5] Classically, it combines a complete or partial triad made of an abdominal muscle deficiency, bilateral cryptorchidism, and dilated urinary tract. The said neonate had abdominal muscle deficiency resulting in hugely distended abdominal wall and dilated urinary tract like enlarged thick walled bladder and dilated and tortuous ureters. But it had ambiguous genitalia with absence of gonads.

Although PBS is associated with other anomalies in $65-70 \%$ of cases and VACTERL is associated with other defects in more than half of cases, the association of VACTERL with PBS is an extremely rare occurrence and only a few cases have been reported till date.[2]

Ozturk, et al reported the first case of concordance of complete Prune belly syndrome and VACTERL. [6] Our patient had vertebral anomalies like kyphosis, anal atresia (imperforate anus), cardiovascular anomalies (enlarged and bilobed heart), renal anomalies like bilateral tortuous ureters with enlarged bladder and limb deformities like talipes equinovarus of right foot. Besides the baby also had deformed thorax with collapsed right lung and hyper- trophied left lung, ambiguous genitalia, enlarged liver and gross asictis. VACTERL association is diagnosed by presence of any three of the classical findings.

Both VACTERL association and PBS have a common etiology of a defect in the differentiating mesoderm occurring between 6th and 10th weeks of gestation.[3] Most of these cases are sporadic. No clear pattern of inheritance has been demonstrated. It is suggested that multiple genetic and environmental factors may play a role in determining the risk of developing and inheriting this disorder. The effects of teratogens, maternal diabetes, in utero exposure to estrogen and/or progesterone containing compounds, statins, lead and infertility treatment have been variably documented as one of the multifactorial causes of VACTERL association. Here the patient has a sibling of 7 years old who is without any abnormality. Even though the mother did not have any other risk factor she was receiving treatment for infertility as she did not conceive during the 7 years since last child birth which might have contributed to this abnormality though not proved.

Prune belly syndrome with VACTERL association have been observed mostly in small for gestational age and usually term infants but the neonate in the above case was normal for gestational age, delivered at 28 weeks of gestation. The mother had ultrasonographic evaluation which detected some of the abnormalities like oligohydramnios of mother grossly distended abdomen of fetus with enlarged and thickened bladder. It had failed to detect the skeletal defects of thorax, vertebral column and right limb, heart and lung anomalies, anal anomalies with ambiguous genitalia which were clearly revealed during detail autopsy examination. And also presence of single umbilical artery (SUA) in the umbilical cord was missed during USG which is an important clue for antenatal diagnosis of VACTERL. The presence of SUA should always result in careful antenatal examination to look for features of VACTERL and other congenital anomalies. Up to $35 \%$ of patients with this anomaly have a single umbilical artery. And this proves advantage of neonatal autopsy over ultrasonography, the 
rate of which is declining gradually at an alarming rate. Therefore the authors express the need for autopsy of every neonatal deaths especially if it is congenitally anomalous.

The prognosis of this association is very poor. It usually depends on degree of renal dysfunction and pulmonary hypoplasia.[3]

Due to debatable etiology and multiple defects in this combination, emphasis is given on early diagnosis and optimal surgical correction. utmost care should be given to every pregnancy and should be thoroughly investigated for any fetal defect. Hence, a correct antenatal diagnosis, proper counseling of parents and earliest treatment in case of any anomalies should be the need of today's practice.

\section{Conclusion}

Prenatal ultrasound plays a mojor role in diagnosing these disorders. Utmost care should be given to every pregnancy and slightest doubt regarding any fetal defect should be thoroughly investigated. A proper counselling of the parents and regular ultrasonographic study in subsequent pregnancies is mandatory.

\section{References}

[1]. Diao B, Diallo Y, Fall PA, Ngom G, Fall B,et al.(2008) Prune -Belly syndrome: Epidemiologic, clinic and therapeutic aspects. Prog Urol.18:470-4.

[2]. Ghritlaharey, Gupta Gourav (2007)Prune belly syndrome associated with incomplete VACTERL,J Indian Assoc Pediatr Surg.12:39-41

[3]. Woods AG, Brandon DH(2007)Prune-Belly syndrome. A focused physical assessment. Adv Neonatal Care;7:132-43.

[4]. Solomon B(2011)D:VACTERL /VATER Association. Orphanet journal Of Rare Diseases;6:56 DOI:10.1186/1750- 1172-6-56 [PMCID:PMC3169446].

[5]. Routh JC, Huang L, Retik AB, Nelson CP(2010) Contemporary epidemiology and characterization of newborn males with Prune -Belly syndrome. Urology;76:44-8.

[6]. Ozturk B, Weber HP, Ganz A(1994) Concordance of Prune-Belly syndrome and VACTERL Association. Klin Padiatr;206:406-9. 